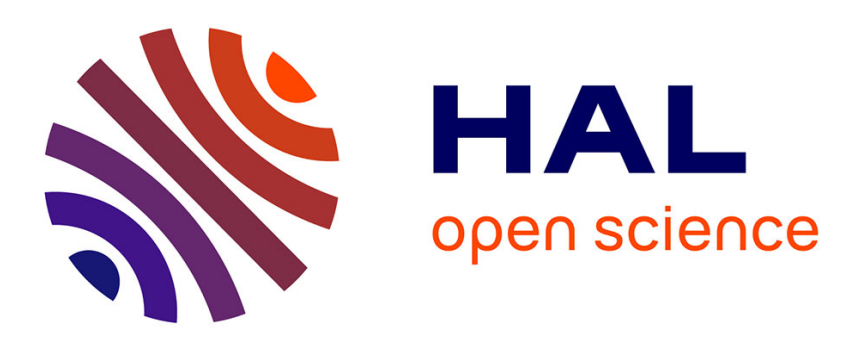

\title{
Sur les actions chimiques de la lumière - (Réponse à l'article de M. A. Guêbhard) \\ P. Villard
}

\section{To cite this version:}

P. Villard. Sur les actions chimiques de la lumière - (Réponse à l'article de M. A. Guêbhard). J. Phys. Theor. Appl., 1905, 4 (1), pp.619-623. 10.1051/jphystap:019050040061901 . jpa-00241039

\section{HAL Id: jpa-00241039 https://hal.science/jpa-00241039}

Submitted on 1 Jan 1905

HAL is a multi-disciplinary open access archive for the deposit and dissemination of scientific research documents, whether they are published or not. The documents may come from teaching and research institutions in France or abroad, or from public or private research centers.
L'archive ouverte pluridisciplinaire HAL, est destinée au dépôt et à la diffusion de documents scientifiques de niveau recherche, publiés ou non, émanant des établissements d'enseignement et de recherche français ou étrangers, des laboratoires publics ou privés. 
VILLARD. - ACTIONŚ CHIMIQUES DE LA LUMIÉRE 619

\section{SUR LES AGTIONS GHIMIQUES DE LA LUMIËRE}

(Réponse à l’article de M. A. Guêbhard);

Par M. P. VILLARD.

Dans un article paru au numéro de mai du Journal de Physique( $\left.{ }^{1}\right)$, M. A. Guébhard a donné un exposé très péremptoire d'une théorie générale qui prétend expliquer tous les phénomènes photographiques. Je crois nécessaire à ce sujet de rectifier les assertions " extraordinaires " et " contradictoires " qu'a bien voulu me prêter M. Guébhard et rétablir l'exactitude des faits que j'avais exposés devant la Société de Physique.

On lit en effet (p. 338) :

"De là les contradictions qui surgissent dès les premières observations faites par Daguerre lui-même, par Claudet, Lerebours et Sécrétan, E. Becquerel, etc., et, en dernier lieu, par M.P. Villard $\left({ }^{2}\right)$, sur l'action dite tantôt continuatrice et tantôt destructrice des verres rouges ou jaunes... "

Et, p. 344:

“ Cependant M. P. Villard assurait que l'effet destructeur (de la lumière jaune, qualifié de continuation pour d'autres substances moins sensibles) subsiste seul. "

J'avouerais volontiers m'être exprimé d'une façon tout à fait inintelligible si je ne supposais pas qu'il est arrivé ici quelque chose d'analogue à ce qui s'est passé dans une circonstance semblable : j'avais montré et décrit (le procès-verbal est exact à ce sujet) un silhouettage blanc, et $M$. Guébhard a expliqué peu de temps après ce phénomène décrit par lui comme un silhouettage noir, plus conforme aux prévisions de la " fonction photographique $\left({ }^{3}\right)$ ".

(1) Voir ce volume p. 334.

(2) Soc. française de Physique, 2 et 16 décembre 1904.

(3) L'explication du silhouettage blanc, ultérieurement donnée par le méme Auteur, est d'ailleurs absolument contraire aux faits. 
Voici exactement ce que j'ai dit (ou du moins voulu dire) :

J'ai fait d'abord de nombreuses expériences avec des verres colorés, les résultats ont été contrôlés et précisés ensuite au spectroscope ('). Voici en quoi ils consistent :

I. Papiers à noircissement direct - non développés - après une impression préalable produisant une grisaille à peine visible, ou mème au-dessous de cette valeur, le papier soumis à l'action d'un spectre donne immédiatement la continuation dans le jaune (maximum entre $\mathrm{D}$ et $\mathrm{E}$ du spectre solaire), tandis qu'une bande témoin sans impression préalable ne donne rigoureusement rien, même dans un temps triple ou quadruple. Le phénomène disparaît si on supprime l'azotate d'argent en excès.

Si l'impression préalable est telle que le papier soit bien visiblement teinté, on constate de plus dans l'extrême rouge et au delà (maximum d'action sur A du spectre) un blanchissement complet du papier sans l'aide d'aucun développement ou lavage $\left({ }^{2}\right)$. Cephénomène n'est sans doute pas compris dans ceux qu'explique la fonction photographique.

II. Plaque au gélatino-chlorure - avec développement alcalin. L'absence complète de tout sel d'argent soluble dans ces plaques supprime tout effet continuateur $\left({ }^{3}\right)$; mais, si on reçoit un spectre sur la plaque préalablement impressionnée, et qu'on développe au bout d'un temps d'exposition que l'on fera varier depuis quelques minutes jusqu'à une limite supérieure ad libilum, on constate que l'impression préalable est progressivement détruite dans le rouge extrême (autour de A solaire) et dans l'infra-rouge jusque vers $\lambda=920$. La sensibilité est complètementrétablie. Dans l'orangé, le jaune, le vert et les autres couleurs, il y a simplement une impression ordinaire plus ou moins forte. Cette impression ne dépasse pas la raie du lithium $(\lambda=670)$; au delà, on n'observe que de la destruction.

III. Gélatino-bromure - développement alcalin. - Le gélatinobromure est beaucoup plus sensible que le chlorure, et sa sensibilité

(1) Ces résultats sont rigoureusement d'accord, jusque dans les détails, avec ceux qu'ont publiés des observateurs tels que Draper, Fizeau et Foucault, Ed. Becquerel.

(2) M. Guébhard affirme (p. 344, en note) que du blanc produirait le même effet. Il est regrettable que M. Guébhard n'ait pas publié d'expériences à ce sujet, car elles seraient fort intéressantes.

(3) Mais, avec les plaques à grain très fin, on obtient cet effet en les imbibant d'azotate d'argent, ou même simplement en les plongeant dans l'eau contenant une trace d'ammoniaque qui dissout un peu du chlorure de la plaque. 
va un peu au delà de la raie du lithium, jusque vers B solaire. Au delà on retrouve le phénomène de destruction précédent.

Bien entendu il faut, surtout avec le gélatino-bromure, atténuer le plus possible la lumière diffusée par le spectroscope et même mettre un verre jaune devant la fente pour supprimer la partie du spectre inutile à l'expérience (bleu et violet).

Ce qui se passe sous les verres colorés est alors facile à prévoir:

Avec les verres jaunes, on a non pas de jaune monochromatique, comme semble l'admettre M. Guébhard, mais toute la partie du spectre comprenant l'infra-rouge, le rouge, l'orangé, le jaune et le jaune vert. Si c'est un papier à azotate d'argent qu'on expose sous ces verres après une légère impression préalable, l'effet continuateur étant le plus rapide (c'est facile à voir au spectroscope), la continuation a le temps de se manifester avant la destruction de l'image latente par le rouge; mais le résultat devient beaucoup meilleur si on ajoute un verre vert $\left({ }^{1}\right)$ (j'avais insisté sur ce point); au contraire, l'addition d'un verre rouge faible $\left({ }^{2}\right)$, qui atténue le jaune, rend l'effet continuateur moins marqué, sans cependant le supprimer; si le verre rouge est très foncé, et surtout si on le double d'un verre bleu, on n'observe plus que la destruction, moins rapide toutefois qu'au spectroscope, parce que le verre rouge a son maximum de transparence dans le rouge orangé et affaiblit l'infra-rouge.

Il n'y a là aucune contradiction. Il n'y en a pas davantage dans le cas du gélatino-chlorure. Sous des verres jaunes qu'on doit superposer en nombre suffisant pour supprimer presque complètement le vert, la plaque est soumise à toutes les radiations, de l'infra-rouge au vert. Comme, en l'absence d'azotate d'argent, il n'y a aucun effet continuateur par le jaune, il ne subsiste que la destruction par le rouge extrême, et l'impression actinique produite par le jaune, impression très faible sur le gélatino-chlorure, et largement compensée par l'action du rouge. Il y a donc destruction de l'image préalable et rien de plus. L'addition d'un verre rouge serait inutile; elle ne donnerait pas plus de rouge que n'en laisse passer le verre jaune; bien au contraire, le verre rouge arrêterait l'infra-rouge plus que ne le fait le verre jaune.

( ${ }^{1}$ Edm. Becquerel fixait entre $D$ et $\mathrm{E}$ le maximum d'effet continuateur et non tantôt dans le rouge, tantôt dans le jaune, comme l'admet M. Guébhàrd.

(2) Les verres rouges laissent passer un peu de bleu; il est nécessaire de les doubler d'un verre jaune, à moins qu'ils ne soient très foncés.

J. de Phys., $4^{\circ}$ série, t. IV. (Septembre 1903.) 
Ainsi, dans cette expérience, on observe uniquement dela destruction produite non pas par du jaune, comme l'admet M. Guébhard, mais par le rouge extrême qui a traversé les verres jaunes, ce que le spectroscope vérifie facilement.

La possibilité de détruire une impression à mesure qu'elle se produit est une conséquence naturelle de ce qui précède et rappelle une expérience d'Ed. Becquerel facile à répéter avec le gélatinochlorure d'argent (et même avec le gélatino-bromure). 11 suffit de prendre une plaque non impressionnée et de l'exposer à un spectre dont au besoin on atténuera un peu le bleu par un faible verre jaune. La lumière diffusée dans l'appareil voile toute la plaque; mais, dans le rouge extrême, ce voile est constamment détruit, et la plaque reste absolument intacte dans cette région, indépendamment du temps de pose, qui peutêtre absolument quelconque.

Voilà donc la superposition de deux impressions qui donne rigoureusement zéro quel que soit le temps de pose, ce qui est en opposition formelle avec les interprétations de M. Guébhard, et d'ailleurs passé sous silence par cet auteur.

Quant au gélatino-bromure, je n'ai jamais dit qu'on pouvait avec lui observer la destruction sous des verres jaunes. J'ai dit au contraire qu'aucun verre coloré ne pouvait convenir, vu qu'aucun ne laisse passer exclusivement l'extrême rouge. C'est seulement au spectroscope que l'expérience peut être faite, et elle est alors très facile à réaliser.

M. Guébhard trouve également contradictoire que la plaque recouvre après destruction sa sensibilité, sous prétexte qu'après destruction de l'action des rayons $\mathrm{X}$ par la lumière blanche elle ne la recouvre pas.

Le cliché que j'ai montré à cette occasion (Soc. de Physique, 16 décembre 1904) n'existerait-il pas? Il y a simplement là un raisonnement incorrect: une plaque impressionnée par la lumière bleue ou violette recouvre sa sensibilité pour cette lumière par le traitement à la lumière rouge; tout ce qu'on pourrait demander à la plaque soumise aux rayons $\mathrm{X}$, puis à la lumière blanche, serait de recouvrer sa sensibilité pour les rayons $X$ et non pour la lumière destructrice. Or ce n'est pas loin d'être exact : on peut faire une bonne radiographie négative ordinaire avec une plaque Jougla traitee: $1^{\circ}$ pendant deux minutes par les rayons $X ; 2^{\circ}$ pendant trente secondes par la lumière directe d'une fenêtre. 
Rien n'oblige d'ailleurs à détruire l'impression par de la lumière blanche, qui n'est pas une couleur; on pent se contenter de prendre le rouge extrême comme pour la lumière; j'ai montré qu'avec les rayons $\mathrm{X}$ la destruction s'étend aussi dans l'infra-rouge. On évitera ainsi la modification que le violet et le bleu font subir au bromure, puisqu'on emploiera une lumière inactinique $\left({ }^{1}\right)$.

En ce qui concerne la théorie de M. Guébhard, je dirai simplement qu'elle ne saurait prétendre à l'explication d'expériences que, de son propre aveu, M. Guébhard a reconnu n'avoir pas répétées. Cet auteur a, par exemple, confondu la continuation avec le phénomène assez explicable de la pose en deux temps avec des lumières actiniques; si, par exemple, une plaque sensible exige à une certaine lumière dix secondes de pose pour donner un commencement d'action, en exposant une partie de la plaque pendant cinq à six secondes seulement, puis la totalité à un voile de même durée, on n'aura d'image que là où les deux impressions sont superposées pour dépasser le seuil de dix secondes. Cette expérience réussit avec toutes les surfaces sensibles, avec toutes les lumières actiniques et avec ces dernières seulement. Elle permet de faire apparaître une image avec une pose réduite à la moitié ou au tiers du seuil. C'est peu de chose comparé à l'effet continuateur, qui équivaut à multiplier la pose par 500 ou 600 , puisque c'est un véritable développement.

Quant aux épreuves si transparentes obtenues avec l'aide des rayons X, épreuves que M. Guébhard considère comme accidentelles, et dues à un " heureux hasard" (ce qui n'est pas un argument), il est extraordinaire qu'après avoir fait tant et de si méthodiques expériences sur la solarisation, l'auteur précédent n'ait jamais pu obtenir cet heureux hasard que j'ai si souvent rencontré. M. Guébhard pourrait-il dire combien il faut de clichés pour que le résultat cesse d'être " accidentel $"\left({ }^{2}\right)$ ?

Dans un autre article, je montrerai que la théorie de la fonction photographique repose uniquement sur des erreurs d'interprétation.

(') J'ai publié dans le Bulletin de la Société d'Encouragement des spectres de destruction complets, comparativement à des spectres de solarisation.

(2) Je ne saurais passer sous silence le singulier argument, d'ailleurs mal fondé, donné par M. Guébhard (p. 343, en note). J'avoue qu'il me paraîtrait excessif de solliciter un prix important pour un procédé qui exige trente secondes de pose. 